\title{
Intersexualität und Transgender: Was FrauenärztInnen darüber wissen sollten
}

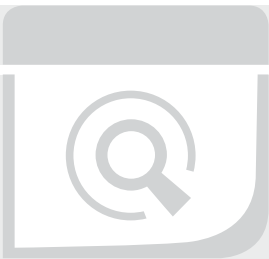

Genderthemen werden aktuell in unserer Gesellschaft öffentlichkeitswirksam und viel diskutiert. Unabhängig davon, ob FrauenärztInnen in ihrer ärztlichen Tätigkeit mit intersexuellen und transsexuellen PatientInnen in Kontakt kommen, besitzt dieses Thema gesamtgesellschaftlich eine hohe Relevanz.

Diese Relevanz spiegelt sich in verschiedenen Bereichen des gesellschaftlichen Lebens wider. So setzen sich u. a. Kunst und Kultur damit auseinander: Filme wie „Tomboy“ oder „Danish Girl“ beschäftigen sich mit nonkonformen Geschlechtsidentitätsentwicklungen im Sinne von Transgenderphänomenen. Zudem konfrontieren uns künstlerisch stilisierte Personen wie Conchita Wurst bewusst mit unseren überholten Ansichten zu binärer Geschlechtsidentität.

Auch in der Modebranche ist das Thema angekommen: H\&M oder Zara kreieren "gender neutral Collections“ und leisten damit einen Beitrag für die Anerkennung nonbinärer Konzepte der Geschlechtsidentität.

Radikale Umbrüche und Paradigmenwechsel zeigen sich im Umgang mit diesen Phänomenen. So sehen wir ein grundlegend verändertes Herangehen der Medizin an die Versorgung von Säuglingen mit intersexuellem Genitale. Diese Entwicklung wurde maßgeblich von AktivistInnen der Betroffenenbewegungen initiiert.

Kinder und Jugendliche beschäftigen sich intensiver und öffentlicher mit ihrer Geschlechtsidentitätsentwicklung (vgl. - Abb. 1). In kinder- und jugendpsychiatrischen Ambulanzen meldet sich eine wachsende Anzahl von Kindern und Jugendlichen, die mit ihrem biologisch zugewiesenen Geschlecht hadern und nach geschlechtsangleichenden Maßnahmen oder einer Hormontherapie fragen.

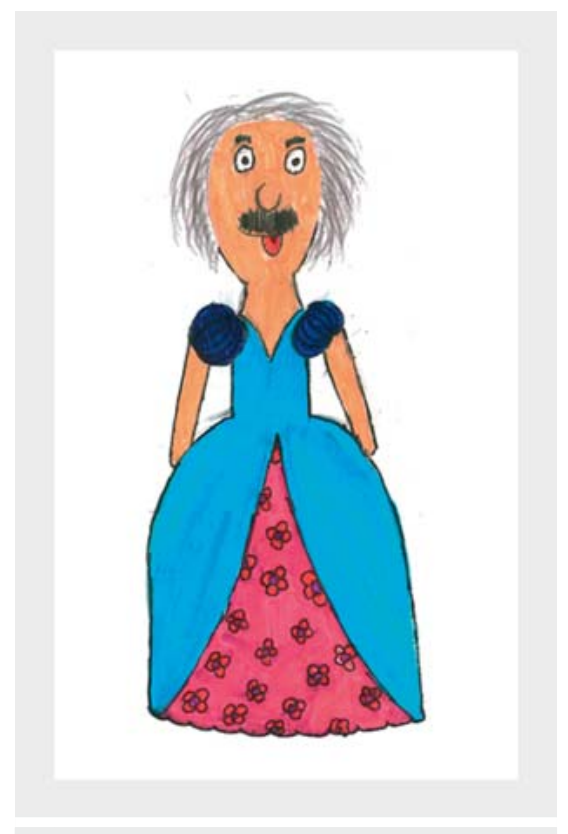

- Abb. 1 Kinderzeichnung „Einstein im Kleid“.

Seit 2010 beschäftigt sich die Bundesregierung mit dem Thema Intersexualität und beauftragte den Deutschen Ethikrat mit einer Stellungnahme. Es gab öffentliche Anhörungen und Diskussionen, die gut dokumentiert sind [1]. 2013 erfolgte eine erste Veränderung des Personenstandsgesetzes (PStG), die es ermöglichte, den Geschlechtseintrag für Neugeborene offen zu lassen. 2018 folgte eine zweite Änderung, die den Eintrag „divers“ ermöglicht. Offensichtlich ist der betroffene Personenkreis jedoch relativ klein. Aktuell bewegen sich die Anträge für den Geschlechtseintrag „divers“ im niedrigen 2-stelligen Bereich pro Jahr [2].
Frauenärztlnnen werden in ihrem klinischen Alltag gelegentlich konfrontiert sein mit Patientinnen, die der Gruppe der Menschen mit intersexueller oder Transgenderidentität zugehörig sind. In diesem Beitrag wird ein Überblick zu den Themen Intersexualität und Transgender gegeben (vgl. - Tab. 1). Zum erstgenannten Themenbereich werden die aktuellen Empfehlungen zum Umgang mit intersexuellen Neugeborenen und der damit verbundenen medizinethischen Debatte dargelegt. Zum zweiten Transgenderthema wird ebenfalls das aktuell empfohlene leitliniengerechte Vorgehen und die kontroverse Debatte dargestellt, die insbesondere die frühe Hormontherapie bei geschlechtsdysphoren Kindern betrifft.

Zuerst ist jedoch ein Überblick über die Begrifflichkeiten erforderlich. In > Tab. 2 sind die derzeitig üblichen Begriffe und deren Erklärungen dargestellt. Diese Übersicht erhebt keinen Anspruch auf Vollständigkeit und ist einem stetigen Wandel unterzogen.

\section{Intersexualität (Varianten der Geschlechtsentwicklung)}

Varianten der sexuellen Entwicklung (DSD = differences of sex development), die wir gemeinhin als Intersexualität bezeichnen, haben vielfältige Ursachen. Relativ bekannt sind zum Beispiel das adrenogenitale Syndrom (AGS), das bei weiblichem Karyotyp $(46, \mathrm{XX})$ infolge von Enzymdefekten zu einer Störung der Steroidsynthese mit Überproduktion von Androgenen und damit einhergehender
- Tab. 1 Gegenüberstellung von Intersexualität und Transgender.

\begin{tabular}{|l|l|}
\hline Intersexualität & Transgender \\
\hline biologisches Geschlecht mehrdeutig & biologisches Geschlecht eindeutig \\
\hline offene Geschlechtsidentitätsentwicklung & $\begin{array}{l}\text { Diskrepanz zwischen biologischem } \\
\text { Geschlecht und Geschlechtsidentität }\end{array}$ \\
\hline
\end{tabular}


\Tab. 2 Wichtige ausgewählte Begriffe [22, 23].

\begin{tabular}{|c|c|}
\hline Begriff & Definition \\
\hline $\begin{array}{l}\text { Genderqueer } \\
\text { Fluid Gender } \\
\text { Non-binary Gender }\end{array}$ & $\begin{array}{l}\text { Geschlechtsidentität, die nicht dichotom zwischen männlich und } \\
\text { weiblich unterscheidet, diese Personen zeigen sowohl männliche als } \\
\text { auch weibliche Attribute der Geschlechtsidentität oder auch keine } \\
\text { Merkmale dieser Geschlechterrollen }\end{array}$ \\
\hline $\begin{array}{l}\text { Geschlechtsdysphorie } \\
\text { (GD) }\end{array}$ & $\begin{array}{l}\text { Unbehagen oder Leiden, das aus der Diskrepanz zwischen der Ge- } \\
\text { schlechtsidentität und dem bei Geburt biologisch zugewiesenen } \\
\text { Geschlecht resultiert (DSM-V-Begriff, der die GIS ablöst) }\end{array}$ \\
\hline Geschlechtsidentität & $\begin{array}{l}\text { internalisierter tief verankerter Sinn für die eigene Geschlechts- } \\
\text { zugehörigkeit als Teil der Identität }\end{array}$ \\
\hline $\begin{array}{l}\text { Geschlechtsidentitäts- } \\
\text { störung (GIS) }\end{array}$ & $\begin{array}{l}\text { veralteter Diagnosebegriff der ICD-10 für Menschen, die sich nicht } \\
\text { mit ihrem biologisch zugewiesenen Geschlecht identifizieren können }\end{array}$ \\
\hline $\begin{array}{l}\text { Geschlechts- } \\
\text { inkongruenz }\end{array}$ & $\begin{array}{l}\text { vorgeschlagener Begriff für die ICD-11 für Menschen, die sich nicht } \\
\text { mit ihrem biologisch zugewiesenen Geschlecht identifizieren können }\end{array}$ \\
\hline $\begin{array}{l}\text { Geschlechts- } \\
\text { nonkonformität }\end{array}$ & $\begin{array}{l}\text { geschlechtlicher Ausdruck der Person unterscheidet sich von den } \\
\text { kulturellen Normen, die von Menschen mit einem bestimmten } \\
\text { Geschlecht erwartet werden }\end{array}$ \\
\hline $\begin{array}{l}\text { Intersexualität } \\
\text { Varianten der Ge- } \\
\text { schlechtsentwicklung }\end{array}$ & $\begin{array}{l}\text { körperliche Geschlechtsmerkmale entsprechen nicht alle einem } \\
\text { Geschlecht, Zwischengeschlechtlichkeit }\end{array}$ \\
\hline LGBTQIA+ & $\begin{array}{l}\text { steht für lesbische, schwule, bisexuelle, transsexuelle, transgender, } \\
\text { queere, intersexuelle und asexuelle Menschen }\end{array}$ \\
\hline Transgender & $\begin{array}{l}\text { Überbegriff für Personen, deren Geschlechtsidentität und -ausdruck } \\
\text { mit dem biologisch vorliegenden Geschlecht differiert }\end{array}$ \\
\hline Transsexualität & $\begin{array}{l}\text { Personen, die sich nicht dem biologisch bei Geburt zugewiesenen } \\
\text { Geschlecht zugehörig fühlen, in der anderen Geschlechterrolle leben } \\
\text { möchten und häufig auch Geschlechtsumwandlungen anstreben } \\
\text { („im falschen Körper gefangen“) }\end{array}$ \\
\hline Transvestitismus & $\begin{array}{l}\text { Tragen der Bekleidung des anderen Geschlechts, unabhängig von der } \\
\text { sexuellen Orientierung, kommt sowohl unter Heterosexuellen als } \\
\text { auch unter Homosexuellen vor }\end{array}$ \\
\hline
\end{tabular}

Virilisierung der weiblichen Neugeborenen einhergeht. Weiterhin gut bekannt sind geschlechtschromosomal bedingte Störungsbilder, wie das Turner-Syndrom $(45, X 0)$ oder das Klinefelter-Syndrom (47, $X X Y)$. Aber auch Störungen der Androgensynthese bei männlichem Karyotyp $(46, X Y)$ gehören dazu. Die Aufzählung ist damit bei Weitem nicht vollständig. Bedeutsam für das weitere Verständnis ist, dass insgesamt sehr verschiedene Störungen zu den Varianten der sexuellen Entwicklung zählen, die mit intersexuellem Genitale einhergehen können.

In den fünfziger Jahren wurde von John Money, einem amerikanischen Sexualwissenschaftler, ein Konzept zum Umgang mit intersexuellen Neugeborenen entwickelt, welches eine frühe Festlegung auf ein Geschlecht beinhaltete und damit einhergehend korrigierende chirurgische In- terventionen [3]. Die betroffenen Eltern wurden dazu angehalten, das Kind in dem zugewiesenen Geschlecht zu erziehen. Eine ganze Reihe dieser betroffenen Kinder haben diese chirurgischen Maßnahmen als traumatisierend erlebt. Weiterhin klagten Betroffene über einen massiven Eingriff in ihr Selbstbestimmungsrecht und ihr Recht auf einen unversehrten Körper. Schließlich lösten gezielte Aktionen der Betroffenenbewegung eine Debatte unter Medizinern und Sexualwissenschaftlern aus, die in Deutschland schließlich in einer Anhörung vor dem Deutschen Ethikrat 2011 mündete und zu weitreichenden Änderungen des per Leitlinien und Konsensuspapieren empfohlenen Vorgehens führte. Frühe operative Eingriffe ohne dringende medizinische Notwendigkeit werden nicht mehr empfohlen. Dagegen wird eine abwartende Haltung präferiert, die es den Betroffenen erlaubt, in einem entsprechenden Alter (ca. 14 Jahre) mit zu entscheiden über etwaige geschlechtsangleichende Maßnahmen. Die Wahrung der Persönlichkeitsrechte und die Entwicklung einer eigenen Geschlechtsidentität sowie die körperliche Unversehrtheit werden deutlich stärker gewichtet. In einem in der renommierten Zeitschrift „Nature Reviews Endocrinology" veröffentlichten Konsensuspapier werden entsprechende Empfehlungen formuliert [4]. Als wichtige Eckpfeiler im Rahmen der psychosozialen Beratung benennen die Autoren die Vermittlung der Problematik als natürliche Variante der menschlichen Entwicklung, die Besprechung der Geschlechtsidentitätsentwicklung unter Zuhilfenahme eines nonbinären Konzepts und die Akzeptanz individueller Entwicklungen. Nicht zuletzt sollen alle nicht dringlichen Interventionen aufgeschoben und mit den Betroffenen in Ruhe besprochen werden. Das betroffene Kind sollte möglichst in einem Alter sein, wo es die Tragweite der Entscheidungen beurteilen kann (ca. 14 Jahre).

Entsprechend dem Konzept einer nonbinären Geschlechtsidentität als Grundlage für die Beratung der betroffenen Familien geht man heutzutage von einem dimensionalen Konstrukt der Geschlechtsidentität aus. Dies beinhaltet nicht nur entweder eine weibliche oder männliche Identität, sondern sieht auch alle möglichen Abstufungen zwischen beiden vor. Demnach ist auch eine intersexuelle Identität denkbar und möglich.

Unter Jugendlichen sind nonbinäre Geschlechtsidentitätsentwicklungen nicht so selten: 1,9 bis $4,6 \%$ zeigen eine ambivalente Geschlechtsidentität (auch als „fluid gender“ oder "genderqueer“ bezeichnet; $[5,6])$. Unter Kindern und Jugendlichen mit einer Transgenderentwicklung ist dieses Phänomen sogar recht häufig mit über $40 \%$ [7]. Diese Tatsache sollte man sich in Anbetracht eventuell gewünschter geschlechtsangleichender Maßnahmen vergegenwärtigen. In der Behandlung von Kindern und Jugendlichen mit Geschlechtsdysphorie sollte mit diesen deshalb auch das Konzept der nonbinären Geschlechtsidentität besprochen und eine voreilige Hormonbehandlung vermieden werden. 


\section{Transgender}

Im zweiten Teil dieses Beitrags folgen Ausführungen zum Themenbereich Transgender und Geschlechtsdysphorie.

Zunächst ein kurzer Einstieg in die Thematik über die Darstellung der Entwicklung der Geschlechtsidentität. Kleinkinder durchleben die Entwicklung ihrer Geschlechtsidentität in Phasen [8]: In der ersten Phase, die etwa mit 11/2 Jahren abgeschlossen ist, bildet sich eine gewisse Geschlechtszugehörigkeit heraus. Das Kind entwickelt eine Überzeugung, männlich oder weiblich zu sein. Die Phase wird deshalb als „gender labeling“ bezeichnet. Anschließend bildet sich eine erste Form der Geschlechtsrollenidentität heraus, die in der 2. Hälfte des 2. Lebensjahres abgeschlossen ist und auch als "gender identity“-Phase bezeichnet wird. Gefolgt wird sie von der Ausgestaltung der eigenen Geschlechtsidentität im Alltag in Form eines geschlechtstypischen Repertoires an Verhalten und Gesten. Es handelt sich um die Phase der "gender role stereotypes“, welche vom 2. bis 3. Lebensjahr und etwas darüber hinaus reicht. Schließlich ist dem Kind zunehmend bewusst, dass die eigene Geschlechtsidentität etwas Bleibendes ist. Diese Phase wird deshalb auch „gender stability“ und im weiteren Verlauf „gender constancy“ genannt. Zu einem vorläufigen ersten Abschluss der Geschlechtsidentitätsentwicklung kommt es im 5. bis 6. Lebensjahr. Zu diesem Zeitpunkt gibt es auch die ersten Vorstellungen von Kindern bei Kinderärzten, die sich dem anderen Geschlecht zugehörig fühlen oder gar im Sinne einer Geschlechtsdysphorie darunter leiden. Betroffene Eltern stellen häufig die Frage, wie sich diese Kinder weiterentwickeln. Ist mit einer transsexuellen oder homosexuellen Entwicklung zu rechnen?

Zunächst einmal muss berücksichtigt werden, dass die Entwicklung der Geschlechtsidentität noch nicht abgeschlossen ist, sondern im Rahmen der Pubertät noch einmal zu einem bestimmenden Thema wird. Die Jugendlichen setzen sich mit der eigenen Geschlechtsidentität intensiv auseinander, sie machen erste sexuelle Erfahrungen und die sexuelle Orientierung bildet sich heraus. Eine ganze Reihe von Wissenschaftlern geht mittlerweile davon aus, dass die Geschlechtsidentitätsentwicklung das ganze Leben über anhält und auch im Erwachsenenalter noch Modifikationen erfährt.

Untersuchungen zur Persistenz der Varianten der Geschlechtsidentitätsentwicklung zeigen, dass eine ganze Reihe der Kinder mit einer sogenannten Geschlechtsidentitätsstörung im jungen Erwachsenenalter eine homosexuelle oder bisexuelle Orientierung aufweisen. In der ersten Untersuchung von Green [9] waren es 75-80\%. In den nachfolgenden Untersuchungen zeigen sich etwas geringere Häufigkeiten mit 50-60\% [10]. Die Geschlechtsidentitätsstörung scheint im Kindesalter eine geringe Persistenz bis ins junge Erwachsenenalter aufzuweisen: Bei Green [9] waren es etwas über $2 \%$. Zucker und Bradley [10] fanden $12 \%$ und die neueren Untersuchungen von Cohen-Kettenis [11] sowie Wallien und Cohen-Kettenis [12] schließlich ca. 16 respektive $27 \%$. Befragt man transidente Jugendliche zu ihrem Festhalten an geschlechtsangleichenden Maßnahmen, so erhält man Häufigkeiten zwischen 43 und $66 \%[10,13,14]$.

Es kann zusammengefasst werden: Je jünger das Kind, desto unsicherer die Prognose bezüglich einer transsexuellen Entwicklung. Damit sollte bei jüngeren Kindern mit Geschlechtsidentitätsproblematik eine abwartende Haltung eingenommen werden [15]. Zudem wünschen bei Weitem nicht alle genderdysphoren Jugendliche geschlechtsangleichende Maßnahmen inklusive einer Hormontherapie, sodass auch bei dieser Gruppe Zurückhaltung und eine kontinuierliche psychologische Begleitung und Beratung erforderlich ist.

In den 90er-Jahren hat sich eine niederländische Forschergruppe um Peggy CohenKettenis intensiv mit der Betreuung und Behandlung von Kindern und Jugendlichen befasst, die mit ihrem biologischen Geschlecht große Probleme hatten und im Sinne einer Geschlechtsidentitätsstörung (überholter Begriff aus der ICD-10) oder Transsexualität eine Geschlechtsumwandlung anstrebten. Die Untersuchungen dieser Gruppe erbrachten Hinweise darauf, dass eine pubertätsbremsende Hormonbehandlung mit GnRH-Analoga die see- lischen Leiden dieser Kinder und Jugendlichen linderte und dass die geschlechtsangleichenden Maßnahmen durch Gabe von gegengeschlechtlichen Sexualhormonen und schließlich geschlechtsangleichende chirurgische Maßnahmen zu einer deutlichen Besserung der seelischen Gesundheit führten $[16,17]$. An dem Behandlungsprotokoll dieser Amsterdamer Klinik [18] orientierten sich im Weiteren die Erstellung verschiedener Leitlinien zur Behandlung dieser Kinder und Jugendlichen (Deutsche Gesellschaft für Kinder und Jugendpsychiatrie - DGKJP, Endocrine Society inklusive Pediatric Endocrine Society, World Professional Association for Transgender Health - WPATH).

Diese Leitlinien empfehlen aktuell etwa folgendes Vorgehen: Zunächst wird eine psychologische Abklärung und Beratung empfohlen, die als Vorbehandlung mindestens 6 Monate dauern sollte und Folgendes beinhaltet:

- diagnostische Einschätzung der Geschlechtsdysphorie und aller anderen gleichzeitig bestehenden psychischen Probleme

- Einschätzung, ob die Geschlechtsdysphorie Ausdruck eines anderen seelischen Problems ist

- Aufklärung über alle Therapieoptionen, Möglichkeiten und Grenzen der Behandlungsmethoden und über den wahrscheinlichen Verlauf der Geschlechtsdysphorie im Kindesalter

- ergebnisoffene und unterstützende Begleitung der Entwicklung des Kindes

- Linderung des Leidensdrucks der Betroffenen und Minderung aller anderen psychosozialen Schwierigkeiten

- Beurteilung der Stabilität der transsexuellen Entwicklung

- beratende Begleitung der Eltern

Die anschließend mögliche erste medizinische Maßnahme ist die Suppression der Pubertätsentwicklung mit GnRH-Analoga, gefolgt von einer weiteren hormonellen Behandlung mit gegengeschlechtlichen Hormonen zur Entwicklung der entsprechenden angestrebten Geschlechtsmerkmale und schließlich chirurgische Maßnahmen zur Geschlechtsangleichung.

Medizinische Maßnahmen sind Kindern und vor allem Jugendlichen vorbehalten, 
die eine möglichst sichere transsexuelle Entwicklung nehmen. Die PatientInnen streben zumeist mit großer Vehemenz nach Maßnahmen, die ihre biologisch angelegten Geschlechtsmerkmale verändern.

Es muss sowohl von jugendlichen PatientInnen als auch deren Eltern oder anderen sorgeberechtigten Bezugspersonen eine Einwilligung zu diesen Maßnahmen eingeholt werden. Eine intensive Aufklärung über realistische Erwartungen, Risiken und Nebenwirkungen, Reversibilität der Maßnahmen und Auswirkungen auf die Fertilität muss erfolgen.

Eine hormonelle Pubertätssuppression gilt als gerechtfertigt, wenn die Diagnose einer Geschlechtsidentitätsstörung respektive einer Geschlechtsdysphorie vorliegt, mindestens Tanner-Stadium 2, und das 12. Lebensjahr erreicht ist und der Pubertätseintritt nachweislich zu einer schweren seelischen Beeinträchtigung führt. Das wichtigste Ziel der Behandlung ist zum einen, die pubertäre Entwicklung und damit die Ausbildung der sekundären Geschlechtsmerkmale zu hemmen, und zum anderen, Zeit zu gewinnen für die weitere Entfaltung der Geschlechtsidentität und die Bewältigung der psychosozialen Herausforderung und Belastungen. Die Behandlung ist vollständig reversibel.

Die nächste Phase der medizinischen Behandlung der Geschlechtsdysphorie ist die Gabe gegengeschlechtlicher Sexualhormone. Diese induziert die Herausbildung der sekundären Geschlechtsmerkmale des angestrebten Geschlechts. Ab etwa dem 16. Lebensjahr wird diese Behandlung empfohlen.

Einige PatientInnen wünschen schließlich chirurgische Maßnahmen zur Geschlechtsangleichung. Diese Maßnahmen sind irreversibel und werden leitliniengemäß erst ab dem 18. Lebensjahr angeboten, abgesehen von der Mastektomie, die auch schon früher möglich erscheint.

Jüngst wurde die S3-Leitlinie zu Geschlechtsinkongruenz, Geschlechtsdysphorie und Transgesundheit für den Erwachsenenbereich veröffentlicht. Federführend wurde sie durch die Deutsche Ge- sellschaft für Sexualforschung (DGfS) unter Beteiligung zahlreicher weiterer Fachgesellschaften erstellt [19]. Zu erwarten ist in Kürze auch eine entsprechende Leitlinie für Kinder und Jugendliche, die für das aktuelle Vorgehen evidenzbasierte Empfehlungen geben wird.

Insbesondere die frühe Hormonbehandlung zur Inhibition der pubertären Entwicklung wird jedoch weiterhin kontrovers diskutiert [20]. Ist es gerechtfertigt, einem 10- oder 12-jährigen Kind pubertätssupprimierende Medikamente zu geben, welches sich in seinem Körper unwohl und nicht richtig fühlt, obwohl die Prognose einer transsexuellen Entwicklung in diesem Alter sehr unsicher ist? Behindert man damit ggf. erste sexuelle Orientierung und Erfahrungen und zementiert damit ggf. iatrogen eine transsexuelle Entwicklung [15]? Diese Fragen sind bis dato unbeantwortet. Eine Linderung des seelischen Leids dieser Kinder und Jugendlichen ist in einfachen Studien mit mäßigem Evidenzgrad nachgewiesen. Qualitativ hochwertige Studien im Sinne von RCTs (randomisierte kontrollierte Studien) stehen aus und sind mit Sicherheit schwierig zu konstruieren und zu organisieren [21].

Rosalia Costa und Kollegen [20] versuchten in einer Überblicksarbeit in der angesehenen Zeitschrift „Nature Reviews Urology“ die Frage zu beantworten: „To treat or not to treat....". Sie beziehen sich damit auf die frühe pubertätssupprimierende Hormonbehandlung. Sie konstatieren Folgendes:

- Wenn das Fortschreiten der Pubertät das psychosoziale Wohlbefinden und die seelische Gesundheit des Kindes gefährdet, dann sollte die Pubertät ausgesetzt werden.

- Psychologische Unterstützung ist essenziell bei der Behandlung der Geschlechtsdysphorie.

- Die Datenlage zu Effekten der GnRHAnaloga ist unzureichend.

- Eine Kosten-Nutzen-Abwägung ist schwierig.

- Es liegen Hinweise für positive Effekte vor.

\section{Fazit}

Abschließend bleibt festzuhalten, dass diverse Ansichten zu Geschlechtsidentität, Intersexualität und der Behandlung von Varianten der sexuellen Entwicklung sowie der Transgenderthematik einem großen Wandel unterworfen sind. Unsere Haltung als MedizinerInnen dazu sollte geprägt sein von Toleranz, Akzeptanz, Offenheit und Respekt gegenüber Ungewohntem, Andersartigen, Fremden.

Zwei recht unterschiedliche Phänomene Intersexualität und Transgender - erfordern gut überlegtes ärztliches Handeln. Neuere Konzepte wie das der nonbinären Geschlechtsidentität können uns dabei helfen, die Betroffenen und deren Eltern gut zu beraten und den Handlungsdruck zu reduzieren.

Es gilt, ärztliches Handeln stets zum Wohle der PatientInnen einzusetzen und nicht zu schaden, getreu der hippokratischen Tradition: „Primum non nocere, secundum cavere, tertium sanare“. Mit manchen Interventionen bei intersexuellen Neugeborenen haben wir in der Vergangenheit dieses wichtige Gebot missachtet, in der Regel jedoch im Glauben, den Eltern und den Betroffenen zu helfen.

Wir neigen als ÄrztInnen gelegentlich dazu, Ansichten von Betroffenen und ihren Initiativen zu belächeln, zu missachten oder gar als Angriff gegen uns selbst zu werten. Es stände uns gut zu Gesicht, diese Haltung zu überwinden und durch mehr Verständnis und Offenheit am Ende adäquatere und vor allem patientengerechtere Hilfe leisten zu können. Wir sind die medizinischen ExpertInnen der Behandlung von unterschiedlichen Erkrankungen. Die betroffenen Patientlnnen, insbesondere jene mit langjährigen Erfahrungen, sind ExpertInnen ihrer Probleme und des Lebens damit im Alltag. Beide Expertisen sind für die Bewältigung von Erkrankungen und Normvarianten der menschlichen Natur von gleichberechtigter Bedeutung.

Die Notwendigkeit eines offenen, vorurteilsfreien und toleranten Umgangs mit allen Spielarten der menschlichen Sexualität gilt gerade auch für das Fachgebiet der Frauenheilkunde und Geburtshilfe als 
einem medizinischen Fachgebiet, welches unmittelbar mit diesen Themen konfrontiert ist.

\section{Interessenkonflikt}

Die Autorinnen/Autoren geben an, dass kein Interessenkonflikt besteht.

\section{Autorinnen/Autoren}

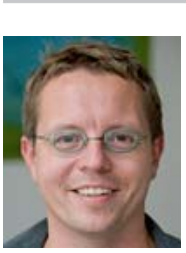

\section{Mirko Döhnert}

PD Dr. med. habil., Klinik und Poliklinik für Psychiatrie, Psychotherapie und Psychosomatik des Kindesund Jugendalters, Universitätsklinikum Leipzig

\section{Korrespondenzadresse}

PD Dr. med. habil. Mirko Döhnert

Oberarzt - Poliklinik und Tagesklinik stv. Klinikdirektor

Universitätsklinikum Leipzig - AöR

Klinik und Poliklinik für Psychiatrie,

Psychotherapie und Psychosomatik

des Kindes- und Jugendalters

Liebigstraße 20a

04103 Leipzig

Mirko.Doehnert@uniklinik-leipzig.de

\section{Literatur}

[1] Deutscher Ethikrat. Dokumentation Intersexualität im Diskurs. 2012. Online: https:// www.ethikrat.org/fileadmin/Publikationen/ Dokumentationen/Diskursdoku_ Intersexualitaet_Druckfassung.pdf; Stand: 25.05 .2020

[2] Sexualität: Geschlechtszuordnung zumeist möglich. Dtsch Arztebl 2020; 117: A-304/ B-270/C-262. Online: https://www. aerzteblatt.de/archiv/212511/SexualitaetGeschlechtszuordnung-zumeist-moeglich; Stand: 25.05.2020

[3] Money J, Hampson JG, Hampson JL. Imprinting and the Establishment of Gender Role. AMA Arch Neurol Psychiatry 1957; 77: 333336
[4] Cools M, Nordenström A, Robeva R et al. COST Action BM1303 working group 1: Caring for individuals with a difference of sex development (DSD): a Consensus Statement. Nat Rev Endocrinol 2018; 14: 415429

[5] Kuyper L, Wijsen C. Gender identities and gender dysphoria in the Netherlands. Arch Sex Behav 2014; 43: 377-385

[6] Pauli D. Nicht-binäre Geschlechtsorientierung bei Kindern und Jugendlichen - Eine Herausforderung für die Betroffenen, das Umfeld und die Behandelnden. Kinderanalyse 2019; 27: 53-64

[7] Veale J, Watson RJ, Adjei J et al. Prevalence of Pregnancy Involvement Among Canadian Transgender Youth and its Relation to Mental Health, Sexual Health, and Gender Identity. Int J Transgend 2016; 17: 107-113

[8] Benjamin J. Phantasie und Geschlecht. Psychoanalytische Studien über Idealisierung, Anerkennung und Differenz. Basel: Verlag Stroemfeld; 1993

[9] Green R, Roberts CW, Williams K et al. Specific cross-gender behaviour in boyhood and later homosexual orientation. $\mathrm{Br}$ J Psychiatry 1987; 151: 84-88

[10] Zucker KJ, Bradley SJ. Gender Identity Disorder and psychosexual Problems in Children and Adolescents. New York: Guilford Press; 1995

[11] Cohen-Kettenis PT. Gender identity disorder in DSM? J Am Acad Child Adolesc Psychiatry 2001; 40: 391

[12] Wallien MSC, Cohen-Kettenis PT. Psychosexual outcome of gender-dysphoric children. J Am Acad Child Adolesc Psychiatry 2008; 47: 1413-1423

[13] Cohen-Kettenis PT, van Goozen SH. Sex reassignment of adolescent transsexuals: a follow-up study. J Am Acad Child Adolesc Psychiatry 1997; 36: 263-271

[14] Smith YL, van Goozen SH, Cohen-Kettenis PT. Adolescents with gender identity disorder who were accepted or rejected for sex reassignment surgery: a prospective followup study. J Am Acad Child Adolesc Psychiatry 2001; 40: 472-481

[15] Döhnert M, Richter-Unruh A, Herrmann E. Geschlechtsdysphorie. Ein Überblick über die aktuelle Studienlage und die kontroverse Diskussion zur Hormontherapie im Kindesund Jugendalter. Kinder- und Jugendmedizin 2018; 18: 190-198
[16] de Vries AL, Steensma TD, Doreleijers TA et al. Puberty suppression in adolescents with gender identity disorder: a prospective follow-up study. J Sex Med 2011; 8: 2276-2283

[17] de Vries AL, McGuire JK, Steensma TD et al. Young adult psychological outcome after puberty suppression and gender reassignment. Pediatrics 2014; 134: 696-704

[18] Delemarre-van de Waal HA, Cohen-Kettenis PT. Clinical management of gender identity disorder in adolescents: A protocol on psychological and paediatric endocrinology aspects. Eur J Endocrinol 2006; 155 (suppl_1): S131-S137

[19] AWMF Leitlinien Geschlechtsinkongruenz, Geschlechtsdysphorie und Trans-Gesundheit: S3-Leitlinie zur Diagnostik, Beratung und Behandlung, AWMF-RegisterNr.138|001. Online: https://www.awmf. org/uploads/tx_szleitlinien/138-001I_S3_ Geschlechtsdysphorie-Diagnostik-BeratungBehandlung_2019-02.pdf; Stand: 25.05.2020

[20] Costa R, Carmichael P, Colizzi M. To treat or not to treat: puberty suppression in childhood-onset gender dysphoria. Nat Rev Urol 2016; 13: 456-462

[21] White Hughto JM, Reisner SL. A Systematic Review of the Effects of Hormone Therapy on Psychological Functioning and Quality of Life in Transgender Individuals. Transgend Health 2016; 1: 21-31

[22] Vance SR, Ehrensaft D, Rosenthal SM. Psychological and medical care of gender nonconforming youth. Pediatrics 2014; 134: 1184-1192

[23] Hembree WC, Cohen-Kettenis PT, Gooren L et al. Endocrine Treatment of Gender-Dysphoric/Gender-Incongruent Persons: An Endocrine Society Clinical Practice Guideline. J Clin Endocrinol Metab 2017; 102: 38693903

\section{Bibliografie}

DOI https://doi.org/10.1055/a-1084-0723 Geburtsh Frauenheilk 2020; 80: 896-900 ๑ Georg Thieme Verlag KG Stuttgart · New York I ISSN 0016-5751 